\title{
Reconstrução de Imagens Tomográficas Discretas em Tomografia Gama Industrial Usando o Algoritmo Variable Neighborhood Search (VNS)
}

\author{
Sylvia Emmanuelle Castelo Branco de Holanda Victor ${ }^{1 *} \quad$ Silvio Soares Bandeira ${ }^{2}$ \\ Emerson Alexandre de Oliveira Lima ${ }^{1}$ \\ ${ }^{1}$ Programa de Pós-Graduação em Engenharia de Sistemas, PPGES-UPE \\ Escola Politécnica da Universidade de Pernambuco \\ Rua Benfica, 455 - Madalena - Recife/PE CEP: 50720-001 \\ ${ }^{2}$ Curso de Ciência da Computação, UNICAP-PE \\ E-mail: sylvia.holanda@gmail.com, silvio@unicap.br, eal@poli.br,
}

\section{RESUMO}

O conceito de tomografia clássica como a visualização das estruturas internas do corpo humano por meio da reconstrução de imagens foi idealizada por W. Oldendorf ([3]) na década de 1960 e fundamentada matematicamente por A. Cormack ([6]). O objetivo era utilizar a radiação para visualizar a estrutura interna do corpo em análise sem que houvesse interferência nas camadas externas deste mesmo, o que explicaria a utilização da técnica de ensaios não destrutivos. Assim, aplicando a atenuação da radiação seria possível atravessar o corpo para obter o perfil de suas densidades.

Vários métodos para processamento de imagens foram desenvolvidos desde então para a reconstrução das imagens dos corpos de prova (também conhecidos como fantomas) de forma cada vez mais rápida, precisa e com menor uso de radiação, como exemplo, segmentação, aprimoramento e medidas das imagens[3,4]. No entanto, um problema notável na reconstrução de imagens em Tomografia Computadorizada é a presença dos ruídos, que em certas aplicações como a médica, impossibilita a identificação precisa do corpo de prova em condições que não sejam estritamente controladas[1].

O projeto de mestrado que foi a base para o desenvolvimento deste presente trabalho apresenta a aplicação do algoritmo metaheurístico VNS (Variable Neighborhood Search), de forma a minimizar os custos com a utilização de poucas trajetórias, ao modelo de reconstrução tomográfica, como um problema de otimização tolerante a presença de ruídos [5]. Diferentemente da tomografia na área médica, os recursos para as medições industriais são bem limitados, de modo que a importância deste trabalho dá-se pela dificuldade computacional de elaborar as reconstruções havendo ruídos. Os resultados apresentados a seguir, são relativos ao primeiro ano do projeto com conclusão prevista para o final do segundo semestre do presente ano [2].

Todo procedimento computacional foi efetuado no MATLAB incluindo a geração de fantomas, cálculo de sua transformada de Radon e o procedimento de reconstrução. A transformada de Radon foi contaminada com ruído branco correspondendo a diferentes níveis de relação sinal e ruído e os algoritmos de reconstrução baseados em VNS foram comparados com aqueles obtidos utilizando a técnica Algebraic Reconstruction Technique[6] (ART). Os valores testados de ruído correspondem, respectivamente, a 1, 2,5 e $10 \%$ do valor absoluto máximo dos dados do sinograma. Foram estudadas reconstruções com 2, 3 e 4 vistas. Os ângulos para o caso de 2 vistas, foram de $0^{\circ}$ e $90^{\circ}$; para o caso de 3 vistas, $0^{\circ}, 60^{\circ}$ e $120^{\circ}$ e para o caso de 4 vistas foram de $0^{\circ}, 60^{\circ}, 90^{\circ}$ e $120^{\circ}$. Para cada vista, foram experimentadas uma quantidade fixa de projeções igualmente espaçadas, a citar, 4,8 e 16 ao todo.

*bolsista de Mestrado com apoio financeiro da Fundação de Amparo à Ciência e Tecnologia do Estado de Pernambuco (Facepe) 
A figura 1 ilustra o comportamento típico das soluções obtidas com relação ao número de trajetórias (quantidade de vistas x quantidade de projeções por vista).

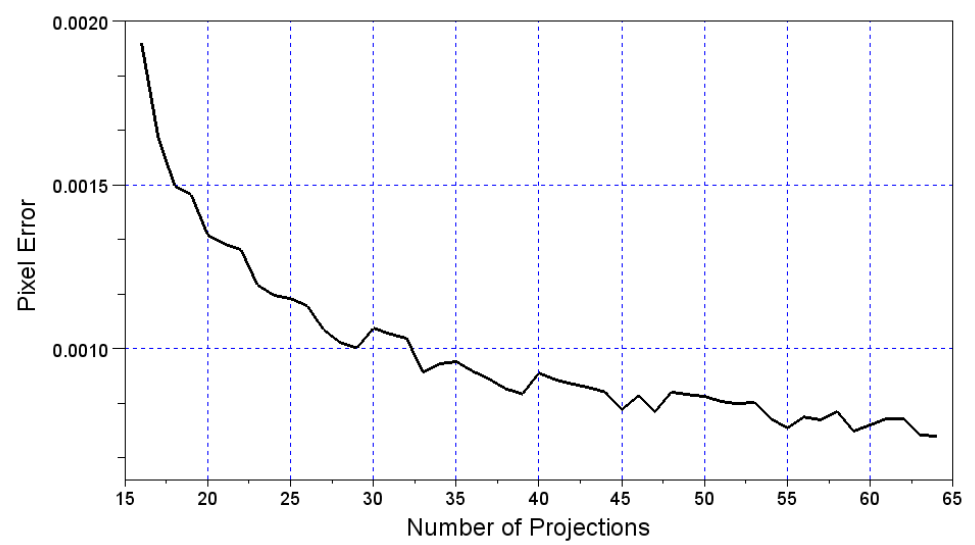

Figura 1: Erro médio quadrático típico entre a imagem reconstruída e a imagem original contaminada por ruído (neste caso de $5 \%$ ) com relação ao número de trajetórias utilizadas. Nesta imagem em particular, o fantoma utilizado consiste em aneis concêntricos de diferentes densidades centrados na origem

Os resultados preliminares desta pesquisa indicam que o algoritmo VNS aplicado para solução do problema da reconstrução tomográfica modelada como um problema de otimização matemática mostrouse bastante eficiente efetuando reconstruções a um baixo custo computacional, com uma pequena quantidade de vistas/projeções e de forma bastante robusta à presença de ruído.

Trabalhos futuros incluem o teste do procedimento na reconstrução de dados reais provenientes de risers de unidades de craqueamento catalítico fluído (FCC) de hidrocarbonetos cuja radiação atenuante utilizada é a radiação gama.

Palavras-chave: Tomografia Industrial, Tomografia Discreta, Tomografia Gama

\section{Referências}

[1] M. Cherfaoui; Innovative Techniques in Non-Destructive Testing and Industrial Applications on Pressure Equipment Procedia Engineering 46:266-278 (2012)

[2] Carlos C. Dantas, Alex Elton Moura, Tiago Leite Rolim, Silvio de Barros Melo, Valdemir Alexandre dos Santos, Emerson Lima. Data Evaluation and Visibility in Single Beam Scanner of Gamma Ray Tomography. World Journal of Nuclear Science and Technology, 2013 vol 3.

[3] A.C. Kak, and M.Slaney, "Principes of computerized tomographic imaging", IEEE Press, New York, 1993.

[4] L. Larrabide, A.A. Novotny, R.A. Feijóo, and R.S.L. Lima, A simple method for tomography reconstruction based on a discrete version of the topological gradient, Inverse Problems, Design and Optimization Symposium (2007).

[5] N. Mladenovic and P. Hansen, Variable Neighbourhood Search. Computers \& Operations Research 113:80-119, (1997).

[6] F. Natterer, "The Mathematics of Computerized Tomography”. SIAM, Philadelphia, 2001. 